\title{
An improvement of convergence rate in the local limit theorem for integral-valued random variables
}

\author{
Tatpon Siripraparat ${ }^{1}$ and Kritsana Neammanee ${ }^{1,2^{*}}$
}

\section{"Correspondence:}

nut.18@hotmail.com;

kritsana.n@chula.ac.th

'Department of Mathematics and

Computer Science, Faculty of

Science, Chulalongkorn University,

10330 Bangkok, Thailand

${ }^{2}$ Commission on Higher Education,

Centre of Excellence in

Mathematics, Sri Ayutthaya, 10400

Bangkok, Thailand

\begin{abstract}
Let $X_{1}, X_{2}, \ldots, X_{n}$ be independent integral-valued random variables, and let $S_{n}=\sum_{j=1}^{n} X_{j}$. One of the interesting probabilities is the probability at a particular point, i.e., the density of $S_{n}$. The theorem that gives the estimation of this probability is called the local limit theorem. This theorem can be useful in finance, biology, etc. Petrov (Sums of Independent Random Variables, 1975) gave the rate $O\left(\frac{1}{n}\right)$ of the local limit theorem with finite third moment condition. Most of the bounds of convergence are usually defined with the symbol O. Giuliano Antonini and Weber (Bernoulli 23(4B):3268-3310, 2017) were the first who gave the explicit constant $C$ of error bound $\frac{C}{\sqrt{n}}$. In this paper, we improve the convergence rate and constants of error bounds in local limit theorem for $S_{n}$. Our constants are less complicated than before, and thus easy to use.
\end{abstract}

MSC: $60 F 05$

Keywords: Local limit theorem; Normal density function; Lattice random variable; Convergence rate

\section{Introduction}

Let $X_{1}, X_{2}, \ldots, X_{n}$ be independent integral-valued random variables with means $\mu_{j}$ and variances $\sigma_{j}^{2}$ for $j=1,2, \ldots, n$. Let $S_{n}=\sum_{j=1}^{n} X_{j}, \mu=\sum_{j=1}^{n} \mu_{j}$, and $\sigma^{2}=\sum_{j=1}^{n} \sigma_{j}^{2}$. One of the interesting probabilities is the probability at a particular point, i.e., $P\left(S_{n}=k\right)$, where $k=1,2, \ldots$. There are two density functions, i.e., discretized normal and normal, to approximate this probability. The discretized normal random variable $\left(\widetilde{Z}_{\mu, \sigma^{2}}\right)$ has the probability mass function

$$
\begin{aligned}
P\left(\widetilde{Z}_{\mu, \sigma^{2}}=k\right) & =P\left(\frac{k-\mu-\frac{1}{2}}{\sigma}<Z_{\mu, \sigma^{2}} \leq \frac{k-\mu+\frac{1}{2}}{\sigma}\right) \\
& =\frac{1}{\sigma \sqrt{2 \pi}} \int_{\frac{k-\mu-\frac{1}{2}}{\sigma}}^{\frac{k-\mu+\frac{1}{2}}{2}} e^{-\frac{x^{2}}{2}} d x,
\end{aligned}
$$

where $Z_{\mu, \sigma^{2}}$ is a normal distribution with mean $\mu$ and variance $\sigma^{2}$.

(c) The Author(s) 2021. This article is licensed under a Creative Commons Attribution 4.0 International License, which permits use sharing, adaptation, distribution and reproduction in any medium or format, as long as you give appropriate credit to the original author(s) and the source, provide a link to the Creative Commons licence, and indicate if changes were made. The images or other third party material in this article are included in the article's Creative Commons licence, unless indicated otherwise in a credit line to the material. If material is not included in the article's Creative Commons licence and your intended use is not permitted by statutory regulation or exceeds the permitted use, you will need to obtain permission directly from the copyright holder. To view a copy of this licence, visit http://creativecommons.org/licenses/by/4.0/. 
To approximate $P\left(S_{n}=k\right)$ by using the discretized normal density function, we can apply the Berry-Esseen theorem. Berry [3] and Esseen [4] were the first two mathematicians who gave the bound between $P\left(S_{n} \leq k\right)$ and the normal distribution. Here is their result.

If $E\left|X_{j}\right|^{3} \leq \infty$ for $j=1,2, \ldots, n$, then

$$
\sup _{k \in \mathbb{R}}\left|P\left(\frac{S_{n}-\mu}{\sigma} \leq k\right)-\frac{1}{\sqrt{2 \pi}} \int_{-\infty}^{k} e^{-\frac{x^{2}}{2}} d x\right| \leq \frac{C_{0}}{\sigma^{3}} \sum_{j=1}^{n} E\left|X_{j}-\mu_{j}\right|^{3}
$$

where $C_{0}$ is an absolute constant.

We can apply (1) to show that

$$
\left|P\left(S_{n}=k\right)-\frac{1}{\sqrt{2 \pi}} \int_{\frac{k-\mu-\frac{1}{2}}{\sigma}}^{\frac{k-\mu+\frac{1}{2}}{2}} e^{-\frac{x^{2}}{2}} d x\right| \leq \frac{2 C_{0}}{\sigma^{3}} \sum_{j=1}^{n} E\left|X_{j}-\mu_{j}\right|^{3} .
$$

The constant $C_{0}$ in (2) was found and improved by many mathematicians (see, [3-10] for examples). The best $C_{0}$ obtained by Shevtsova [8] in 2013 was 0.5583 for the case of non-identically and 0.469 for the case of identically.

The local limit theorem describes how the probability mass function of a sum of independent discrete random variables approaches the normal density.

Let

$$
\epsilon_{n}(k)=\left|P\left(S_{n}=k\right)-\frac{1}{\sigma \sqrt{2 \pi}} e^{-\frac{(k-\mu)^{2}}{2 \sigma^{2}}}\right| .
$$

De Moivre and Laplace (see [11]) established the local limit theoremDe Moivre and Laplace (see [11]) established the local limit theorem for the binomial case in 1754. For sums of independent random variables, we can prove the local limit theorem by using the Berry-Esseen theorem and get the rate convergence $O\left(\frac{1}{\sqrt{n}}\right)$ (see [2]).

In 1971, Ibragimov and Linnik improved the rate of convergence from $O\left(\frac{1}{\sqrt{n}}\right)$ to $O\left(\frac{1}{n^{\frac{1}{2}+\alpha}}\right)$, $0<\alpha<\frac{1}{2}$, in the case of $X_{j}$ s being identical and square integrable random variables.

For the non-identical case, Petrov $(1975,[1])$ showed that if

$1 \sigma^{2} \rightarrow \infty$ as $n \rightarrow \infty$,

$2 \sum_{j=1}^{n} E\left|X_{j}-\mu_{j}\right|^{3}=O\left(\sigma^{2}\right)$,

$3 P\left(X_{j}=0\right) \geq P\left(X_{j}=m\right)$ for all $j$ and $m$ and

$4 \operatorname{gcd}\left\{m: \frac{1}{\log n} \sum_{j=1}^{n} P\left(X_{j}=0\right) P\left(X_{j}=m\right) \rightarrow \infty\right.$ as $\left.n \rightarrow \infty\right\}=1$,

then

$$
\epsilon_{n}(k) \leq \frac{C_{1}}{\sigma^{2}} .
$$

Furthermore, Petrov ([1], see also [2]) improved the rate of convergence from $O\left(\frac{1}{\sigma^{2}}\right)$ to $O\left(\frac{1}{n \sqrt{n}}\right)$ in the case of a symmetric binomial.

In the previous studies, no one gave the explicit constants of error bounds. Most of the theorems were usually presented in the form of $O$. Therefore, finding the constants has been interesting. In 2018, Zolotukhin, Nagaev, and Chebotarev [12] gave the convergence with a constant of error bound in the case that $S_{n}$ is a binomial. They showed that

$$
\epsilon_{n}(k) \leq \min \left\{\frac{1}{\sigma \sqrt{2 e}}, \frac{0.516}{\sigma^{2}}\right\}
$$


After that Siripraparat and Neammanee [13] relaxed the identically condition and obtained the convergence in the case of Poisson binomial in 2020. Their result is

$$
\epsilon_{n}(k) \leq \frac{0.1194}{\sigma^{2}\left(1-\frac{3}{4 \sigma}\right)^{3}}+\frac{0.0749}{\sigma^{3}}+\frac{0.2107}{\sigma^{3}\left(1-\frac{3}{4 \sigma}\right)^{6}}+\left(\frac{0.4579}{\sqrt{\sigma}}+\frac{0.4725}{\sigma \sqrt{\sigma}}\right) e^{-\frac{3 \sigma}{2}} .
$$

Furthermore, in the case of $S_{n}=\operatorname{Bi}\left(\frac{1}{2}\right)$ being a symmetric binomial, i.e., $P\left(X_{j}=1\right)=\frac{1}{2}=$ $1-P\left(X_{j}=0\right)$, they showed that

$$
\epsilon_{n}(k) \leq \frac{0.5992}{n \sqrt{n}}+\frac{3.3984}{n^{2}\left(1-\frac{3}{2 \sqrt{n}}\right)^{4}}+\frac{337.8048}{n^{3} \sqrt{n}\left(1-\frac{3}{2 \sqrt{n}}\right)^{8}}+\left(\frac{0.6476}{n^{\frac{1}{4}}}+\frac{1.3365}{n^{\frac{3}{4}}}\right) e^{-\frac{3 \sqrt{n}}{4}} .
$$

In 2017, Giuliano Antonini and Weber [2] gave the rate of convergence $O\left(\frac{1}{\sigma}\right)$ with a constant of error bound in the case of sums of independent lattice random variables. $X$ is a lattice random variable when the value of $X$ is in $L(a, b)=\left\{v_{k}\right\}$, where $v_{k}=a+b k, k \in \mathbb{Z}$, $a$ and $b>0$ are real numbers. They gave the following theorem.

Theorem 1.1 (See [2]) Let $X_{1}, X_{2}, \ldots, X_{n}$ be independent square integrable random variables taking values in a lattice $L(a, b)$ and $S_{n}=\sum_{j=1}^{n} X_{j}$. Let $\alpha_{X}=\sum_{k \in \mathbb{Z}} \min \left\{P\left(X=v_{k}\right), P(X=\right.$ $\left.\left.v_{k+1}\right)\right\}$ and $V_{j} s, L_{j} s, \epsilon_{j}$ s be such that

$$
V_{j}+\epsilon_{j} b L_{j} \stackrel{D}{=} X_{j} \quad \text { for all } j=1,2, \ldots, n,
$$

where $P\left(L_{j}=0\right)=P\left(L_{j}=1\right)=\frac{1}{2}, P\left(\epsilon_{j}=1\right)=1-P\left(\epsilon_{j}=0\right)=q_{j}$, where $0<q_{j} \leq \alpha_{X_{j}}$ for all $j=1,2, \ldots, n$, and $\left(V_{j}, \epsilon_{j}\right)$ and $L_{j}$ are independent for each $j=1,2, \ldots, n$.

\section{Assume that}

$1 \frac{\log \lambda_{n}}{\lambda_{n}} \leq \frac{1}{14}$, where $\lambda_{n}=\sum_{j=1}^{n} q_{j}$

$2 \frac{\left(k-E S_{n}\right)^{2}}{\operatorname{Var}\left(S_{n}\right)} \leq\left(\frac{\lambda_{n}}{14 \log \lambda_{n}}\right)^{\frac{1}{2}}$ for all $k \in L(n a, b)$.

Then

$$
\left|P\left(S_{n}=k\right)-\frac{b}{\sqrt{2 \pi \operatorname{Var}\left(S_{n}\right)}} e^{-\frac{\left(k-E S_{n}\right)^{2}}{2 \operatorname{Var} S_{n}}}\right| \leq C_{2}\left[b\left(\frac{\log \lambda_{n}}{\operatorname{Var}\left(S_{n}\right) \lambda_{n}}\right)^{\frac{1}{2}}+\frac{\delta_{n}+\lambda_{n}^{-1}}{\sqrt{\lambda_{n}}}\right],
$$

where

$$
\begin{aligned}
& C_{2}=2^{\frac{7}{2}} \max \left\{\frac{8}{\sqrt{2 \pi}}, C_{3}\right\}, \\
& C_{3} \text { is the constant such that } \sup _{z}\left|P\left(\operatorname{Bi}\left(\frac{1}{2}\right)=z\right)-\sqrt{\frac{2}{\pi n}} e^{-\frac{(2 z-n)^{2}}{2 n}}\right| \leq \frac{C_{3}}{n \sqrt{n}}, \\
& \delta_{n}=\sup _{x \in \mathbb{R}}\left|P\left(\frac{S_{n}^{\prime}-E S_{n}^{\prime}}{\sqrt{\operatorname{Var}\left(S_{n}^{\prime}\right)}}<x\right)-P\left(Z_{0,1}<x\right)\right|, \quad \text { and } \\
& S_{n}^{\prime}=W_{n}+\frac{b}{2} B_{n}, W_{n}=\sum_{j=1}^{n} V_{j} \text { and } B_{n}=\sum_{j=1}^{n} \epsilon_{j} .
\end{aligned}
$$

Note that if we choose the constant of error bound $C_{3}$ in (5), then $C_{2}$ is 36.1082 and the rate of Theorem 1.1 is $O\left(\frac{1}{\sigma}\right)$. We can see that the bound of [2] depends on $C_{3}$ and is still 
complicated. In this work, we improve the rate of convergence of [2] to be $O\left(\frac{1}{\sigma^{2}}\right)$ and also give the constant of error bound. Our constants are not complicated and can be applied easily. The results are shown in the following.

Theorem 1.2 Let $X_{1}, X_{2}, \ldots, X_{n}$ be independent integral-valued random variables and $\alpha_{j}=$ $2 \sum_{l=-\infty}^{\infty} p_{j l} p_{j(l+1)}$, where $p_{j l}=P\left(X_{j}=l\right)$. If $\alpha_{j}>0$ for all $j=1,2, \ldots, n$, then

$$
\epsilon_{n}(k) \leq \frac{2.2075 e^{-\frac{\tau^{2} \alpha}{\pi^{2}}}}{\tau \alpha}+\frac{1.7898}{\sigma^{4}} \sum_{j=1}^{n} E\left|X_{j}\right|^{3},
$$

where $\tau=\frac{1}{10 \sqrt[3]{\sum_{j=1}^{n} E\left|X_{j}\right|^{3}}}$ and $\alpha=\sum_{j=1}^{n} \alpha_{j}$.

$b$ is said to be maximal when there are no other numbers $a^{\prime}$ and $b^{\prime}>b$ for which $P(X \in$ $\left.L\left(a^{\prime}, b^{\prime}\right)\right)=1$.

Theorem 1.3 Let $X_{1}, X_{2}, \ldots, X_{n}$ be independent random variables in a maximal lattice $L(a, b)$ and

$$
\delta_{n}(k)=\left|P\left(S_{n}=n a+k b\right)-\frac{b}{\sigma \sqrt{2 \pi}} e^{-\frac{(b(n a+k b)-(\mu-n a))^{2}}{2 \sigma^{2}}}\right| .
$$

Then

$$
\delta_{n}(k) \leq \frac{2.2075 e^{-\frac{\tau^{2} \alpha}{\pi^{2}}}}{\tau \alpha}+\frac{1.7898 b^{4}}{\sigma^{4}} \sum_{j=1}^{n} E\left|X_{j}\right|^{3},
$$

where $\alpha_{j}=2 \sum_{l=-\infty}^{\infty} p_{j l} p_{j(l+1)}, p_{j l}=P\left(X_{j}=a+b l\right)$, and $\alpha=\sum_{j=1}^{n} \alpha_{j}$.

Theorem 1.4 If $X_{1}, X_{2}, \ldots, X_{n}$ in Theorem 1.3 are independent identically distributed (i.i.d.), then

$$
\delta_{n}(k) \leq \frac{2.2075 e^{-\frac{\tau^{2} \alpha}{\pi^{2}}}}{\tau \alpha}+\frac{1.7898 b^{4}}{n \sigma_{1}^{4}} E\left|X_{1}\right|^{3},
$$

where $\tau=\frac{1}{10 \sqrt[3]{n E\left|X_{1}\right|^{3}}}$ and $\alpha=2 n \sum_{l=-\infty}^{\infty} p_{l} p_{l+1}, p_{l}=P\left(X_{1}=a+b l\right)$.

Observe that the constant in Theorems 1.2-1.4 is easier than the constant in Theorem 1.1.

We organize this paper as follows. In Sect. 2, we give the exponential bounds of a characteristic function which will be used to prove the main theorems in Sect. 3. After that we give some examples in Sect. 4.

\section{Exponential bounds of a characteristic function}

In this section, we let $X$ be an integral-valued random variable with characteristic function $\psi$ and $\theta(t)=$ argument of $\psi(t)$. Then

$$
\psi(t)=\sum_{j=-\infty}^{\infty} p_{j} e^{i j t}, \quad \text { where } p_{j}=P(X=j) \text { for } t \in \mathbb{R}
$$


and

$$
\theta(t)=\arctan \left(\frac{\sum_{j=-\infty}^{\infty} p_{j} \sin (j t)}{\sum_{j=-\infty}^{\infty} p_{j} \cos (j t)}\right) .
$$

Characteristic functions are important in probability theory and statistics, especially in local limit theorems, stability problems, etc. In the study of local limit theorems, it is required to estimate the bounds for modulus $|\psi(t)|$ of a characteristic function $\psi$. The various bounds for $|\psi(t)|$ play a key role in the investigation of the rate of convergence in the local limit theorems. Previous studies have shown the bounds for $|\psi(t)|$ in the case of continuous and bounded random variable in a variety of versions (see [14-18] for example). In addition, the bounds for $|\psi(t)|$ of a lattice random variable have been shown in a number of research works (see [18-21] for example). Furthermore, there is the exponential bound for $|\psi(t)|$ of a Poisson binomial distribution as shown in Neammanee [22]. In this section, we use the idea of Neammanee [22] to obtain the exponential bound for $|\psi(t)|$ of an integral-valued random variable. The following lemmas are our results.

Lemma 2.1 Let $t \in[0, \pi)$ and $\alpha=2 \sum_{j=-\infty}^{\infty} p_{j} p_{j+1}$. Then $|\psi(t)| \leq e^{-\frac{1}{\pi^{2}} \alpha t^{2}}$.

Proof Let $t \in[0, \pi)$. If $|\psi(t)|=0$, then Lemma 2.1 holds. Assume that $|\psi(t)|>0$.

Note that

$$
\begin{aligned}
|\psi(t)|^{2} & =\psi(t) \overline{\psi(t)} \\
& =\sum_{j=-\infty}^{\infty} p_{j} e^{i j t} \sum_{l=-\infty}^{\infty} p_{l} e^{-i l t} \\
& =\sum_{j=-\infty}^{\infty} \sum_{l=-\infty}^{\infty} e^{i t(j-l)} p_{j} p_{l} \\
& =\sum_{j=-\infty}^{\infty} \sum_{l=-\infty}^{\infty} \cos ((j-l) t) p_{j} p_{l}+i \sum_{j=-\infty}^{\infty} \sum_{l=-\infty}^{\infty} \sin ((j-l) t) p_{j} p_{l} .
\end{aligned}
$$

Since $|\psi(t)|^{2}$ is real, we get

$$
\begin{aligned}
|\psi(t)|^{2} & =\sum_{j=-\infty}^{\infty} \sum_{l=-\infty}^{\infty} \cos ((j-l) t) p_{j} p_{l} \\
& =\sum_{j=-\infty}^{\infty} \sum_{l=-\infty}^{\infty}\left(1-2 \sin ^{2}\left((j-l) \frac{t}{2}\right)\right) p_{j} p_{l} \\
& =\sum_{j=-\infty}^{\infty} \sum_{l=-\infty}^{\infty} p_{j} p_{l}-2 \sum_{j=-\infty}^{\infty} \sum_{l=-\infty}^{\infty} \sin ^{2}\left((j-l) \frac{t}{2}\right) p_{j} p_{l} \\
& =1-2 \sum_{j=-\infty}^{\infty} \sum_{l=-\infty}^{\infty} \sin ^{2}\left((j-l) \frac{t}{2}\right) p_{j} p_{l} .
\end{aligned}
$$


From this fact and the fact that $|\psi(t)|>0$, we have

$$
0 \leq 2 \sum_{j=-\infty}^{\infty} \sum_{l=-\infty}^{\infty} \sin ^{2}\left((j-l) \frac{t}{2}\right) p_{j} p_{l}<1
$$

By (7) and (8), we get

$$
\begin{aligned}
\ln |\psi(t)| & =\frac{1}{2} \ln \left(1-2 \sum_{j=-\infty}^{\infty} \sum_{l=-\infty}^{\infty} \sin ^{2}\left((j-l) \frac{t}{2}\right) p_{j} p_{l}\right) \\
& =-\frac{1}{2} \sum_{k=1}^{\infty} \frac{1}{k}\left[2 \sum_{j=-\infty}^{\infty} \sum_{l=-\infty}^{\infty} \sin ^{2}\left((j-l) \frac{t}{2}\right) p_{j} p_{l}\right]^{k} \\
& \leq-\sum_{j=-\infty}^{\infty} \sum_{l=-\infty}^{\infty} \sin ^{2}\left((j-l) \frac{t}{2}\right) p_{j} p_{l} \\
& =-\sum_{j=-\infty}^{\infty} \sum_{l=-\infty}^{\infty} \sin ^{2}\left(|j-l| \frac{t}{2}\right) p_{j} p_{l} \\
& \leq-\sum_{j=-\infty}^{\infty} \sum_{l=-\infty}^{\infty} \frac{(j-l)^{2} t^{2}}{\pi^{2}} p_{j} p_{l} \\
& =-\frac{1}{\pi^{2}} \alpha t^{2},
\end{aligned}
$$

where we use the fact that $\sin \left(\frac{t}{2}\right) \geq \frac{t}{\pi}$ on $[0, \pi)$ in the last inequality.

Hence, $|\psi(t)| \leq e^{-\frac{1}{\pi^{2}} \alpha t^{2}}$.

Lemma 2.2 For $t \in[0, \pi]$,

$$
|\psi(t)| \leq e^{-\frac{1}{2} \sigma^{2}(X) t^{2}+\frac{2}{3} E|X|^{3} t^{3}} .
$$

Proof The lemma holds if $|\psi(t)|=0$. Assume that $|\psi(t)|>0$.

Note that

$$
\begin{aligned}
\sum_{j=-\infty}^{\infty} \sum_{l=-\infty}^{\infty}(j-l)^{2} p_{j} p_{l} & =\sum_{j=-\infty}^{\infty} \sum_{l=-\infty}^{\infty} \sum_{m=0}^{2}\left(\begin{array}{l}
2 \\
m
\end{array}\right) j^{m}(-l)^{2-m} p_{j} p_{l} \\
& =\sum_{m=0}^{2}(-1)^{2-m}\left(\begin{array}{c}
2 \\
m
\end{array}\right) \sum_{j=-\infty}^{\infty} j^{m} p_{j} \sum_{l=-\infty}^{\infty} l^{2-m} p_{l} \\
& =\sum_{m=0}^{2}(-1)^{2-m}\left(\begin{array}{c}
2 \\
m
\end{array}\right) E X^{m} E X^{2-m} \\
& =E X^{2}-2(E X)^{2}+E X^{2} \\
& =2 \sigma^{2}(X)
\end{aligned}
$$


and

$$
\sum_{j=-\infty}^{\infty} \sum_{l=-\infty}^{\infty}(|j|+|l|)^{3} p_{j} p_{l} \leq 4 \sum_{j=-\infty}^{\infty} \sum_{l=-\infty}^{\infty}\left(|j|^{3}+|l|^{3}\right) p_{j} p_{l}=8 E|X|^{3},
$$

where we use the fact that $(a+b)^{k} \leq 2^{k-1}\left(a^{k}+b^{k}\right), a, b \geq 0$, and $k \in \mathbb{N}$ in the first inequality.

From the fact that

$$
\cos (a t)=1-\frac{1}{2} a^{2} t^{2}+\frac{1}{6} a^{3} t^{3} \sin \left(t_{1}\right) \quad \text { for some } t_{1}
$$

and (9), (10), (11), we get

$$
\begin{aligned}
\ln |\psi(t)| & \\
\leq & -\sum_{j=-\infty}^{\infty} \sum_{l=-\infty}^{\infty} \sin ^{2}\left((j-l) \frac{t}{2}\right) p_{j} p_{l} \\
& =-\sum_{j=-\infty}^{\infty} \sum_{l=-\infty}^{\infty}\left[\frac{1}{2}-\frac{1}{2} \cos ((j-l) t)\right] p_{j} p_{l} \\
& =-\sum_{j=-\infty}^{\infty} \sum_{l=-\infty}^{\infty}\left[\frac{1}{2}-\frac{1}{2}\left(1-\frac{1}{2}(j-l)^{2} t^{2}+\frac{1}{6}(j-l)^{3} t^{3} \sin \left((j-l) t_{1}\right)\right)\right] p_{j} p_{l} \\
& =-\sum_{j=-\infty}^{\infty} \sum_{l=-\infty}^{\infty}\left[\frac{1}{4}(j-l)^{2} t^{2}-\frac{1}{12}(j-l)^{3} t^{3} \sin \left((j-l) t_{1}\right)\right] p_{j} p_{l} \\
& \leq-\frac{t^{2}}{4} \sum_{j=-\infty}^{\infty} \sum_{l=-\infty}^{\infty}(j-l)^{2} p_{j} p_{l}+\frac{t^{3}}{12} \sum_{j=-\infty}^{\infty} \sum_{l=-\infty}^{\infty}|j-l|^{3} p_{j} p_{l} \\
& \leq-\frac{1}{2} \sigma^{2}(X) t^{2}+\frac{t^{3}}{12} \sum_{j=-\infty}^{\infty} \sum_{l=-\infty}^{\infty}(|j|+|l|)^{3} p_{j} p_{l} \\
& \leq-\frac{1}{2} \sigma^{2}(X) t^{2}+\frac{2}{3} E|X|^{3} t^{3} .
\end{aligned}
$$

Hence, $|\psi(t)| \leq e^{-\frac{1}{2} \sigma^{2}(X) t^{2}+\frac{2}{3} E|X|^{3} t^{3}}$.

Lemma 2.3 Let $\tau_{1}=\frac{1}{10 \sqrt[3]{E|X|^{3}}}$. Then

$$
|\psi(t)| \geq e^{-\frac{1}{2} \sigma^{2}(X) t^{2}-\frac{2}{3} E|X|^{3} t^{3}} \quad \text { for } t \in\left[0, \tau_{1}\right] .
$$

Proof Since $|\sin (\theta)| \leq|\theta|$ and (10),

$$
2 \sum_{j=-\infty}^{\infty} \sum_{l=-\infty}^{\infty} \sin ^{2}\left((j-l) \frac{t}{2}\right) p_{j} p_{l} \leq \frac{t^{2}}{2} \sum_{j=-\infty}^{\infty} \sum_{l=-\infty}^{\infty}(j-l)^{2} p_{j} p_{l}=t^{2} \sigma^{2}(X)
$$

Note that

$$
\sigma^{2}(X) \leq E\left(X^{2}\right) \leq\left(E|X|^{3}\right)^{\frac{2}{3}} .
$$


From (12), (13), and the fact that $t^{2} \leq \frac{1}{100\left(E|X|^{3}\right)^{\frac{2}{3}}}$,

$$
0 \leq 2 \sum_{j=-\infty}^{\infty} \sum_{l=-\infty}^{\infty} \sin ^{2}\left((j-l) \frac{t}{2}\right) p_{j} p_{l} \leq \frac{1}{100}
$$

Therefore,

$$
1-2 \sum_{j=-\infty}^{\infty} \sum_{l=-\infty}^{\infty} \sin ^{2}\left((j-l) \frac{t}{2}\right) p_{j} p_{l} \geq \frac{99}{100}
$$

and

$$
-\frac{1}{1-2 \sum_{j=-\infty}^{\infty} \sum_{l=-\infty}^{\infty} \sin ^{2}\left((j-l) \frac{t}{2}\right) p_{j} p_{l}} \geq-\frac{100}{99}
$$

By (9), (12), (13), and (15), we get

$$
\begin{aligned}
\ln |\psi(t)|= & -\sum_{j=-\infty}^{\infty} \sum_{l=-\infty}^{\infty} \sin ^{2}\left((j-l) \frac{t}{2}\right) p_{j} p_{l} \\
& -\frac{1}{2} \sum_{k=2}^{\infty} \frac{1}{k}\left[2 \sum_{j=-\infty}^{\infty} \sum_{l=-\infty}^{\infty} \sin ^{2}\left((j-l) \frac{t}{2}\right) p_{j} p_{l}\right]^{k} \\
\geq & -\frac{1}{2} \sigma^{2}(X) t^{2}-\frac{1}{4} \frac{\left[2 \sum_{j=-\infty}^{\infty} \sum_{l=-\infty}^{\infty} \sin ^{2}\left((j-l) \frac{t}{2}\right) p_{j} p_{l}\right]^{2}}{2 \sum_{j=-\infty}^{\infty} \sum_{l=-\infty}^{\infty} \sin ^{2}\left((j-l) \frac{t}{2}\right) p_{j} p_{l}} \\
\geq & -\frac{1}{2} \sigma^{2}(X) t^{2}-\frac{1}{4}\left(\frac{100}{99}\right)\left[2 \sum_{j=-\infty}^{\infty} \sum_{l=-\infty}^{\infty} \sin ^{2}\left((j-l) \frac{t}{2}\right) p_{j} p_{l}\right]^{2} \\
\geq & -\frac{1}{2} \sigma^{2}(X) t^{2}-\frac{25}{99} \sigma^{4}(X) t^{4} \\
\geq & -\frac{1}{2} \sigma^{2}(X) t^{2}-\frac{25}{99}\left(E|X|^{3}\right)^{\frac{4}{3}} t^{4} \\
\geq & -\frac{1}{2} \sigma^{2}(X) t^{2}-\frac{25}{99}\left(E|X|^{3}\right)^{\frac{4}{3}} \frac{1}{10 \sqrt[3]{E|X|^{3}}} t^{3} \\
\geq & -\frac{1}{2} \sigma^{2}(X) t^{2}-\frac{2}{3} E|X|^{3} t^{3} .
\end{aligned}
$$

Hence, $|\psi(t)| \geq e^{-\frac{1}{2} \sigma^{2}(X) t^{2}-\frac{2}{3} E|X|^{3} t^{3}}$ for $t \in\left[0, \tau_{1}\right]$.

\section{Lemma 2.4}

$1 \theta^{(1)}(0)=E X$.

$2 \theta^{(2)}(0)=0$.

$3\left|\theta^{(3)}(t)\right| \leq 4.2874 E|X|^{3}$ for $t \in\left[0, \tau_{1}\right]$.

Proof 1. By (6), we get

$$
\theta^{(1)}(0)=\frac{\sum_{j=-\infty}^{\infty} \sum_{l=-\infty}^{\infty} j p_{j} p_{l}}{\sum_{j=-\infty}^{\infty} \sum_{l=-\infty}^{\infty} p_{j} p_{l}}=E X .
$$


2. Let $A(t)=\sum_{j=-\infty}^{\infty} \sum_{l=-\infty}^{\infty} j \cos ((j-l) t) p_{j} p_{l}$ and $B(t)=\sum_{j=-\infty}^{\infty} \sum_{l=-\infty}^{\infty} \cos ((j-l) t) p_{j} p_{l}$. Observe that

$$
\theta^{(1)}(t)=\frac{A(t)}{B(t)} \quad \text { and } \quad \theta^{(2)}(t)=\frac{B(t) A^{\prime}(t)-A(t) B^{\prime}(t)}{(B(t))^{2}},
$$

where

$$
\begin{aligned}
& A^{\prime}(t)=-\sum_{j=-\infty}^{\infty} \sum_{l=-\infty}^{\infty} j(j-l) \sin ((j-l) t) p_{j} p_{l} \quad \text { and } \\
& B^{\prime}(t)=-\sum_{j=-\infty}^{\infty} \sum_{l=-\infty}^{\infty}(j-l) \sin ((j-l) t) p_{j} p_{l} .
\end{aligned}
$$

Since $A^{\prime}(0)=0$ and $B^{\prime}(0)=0, \theta^{(2)}(0)=0$.

3. Note that

$$
|A(t)|=\left|\sum_{j=-\infty}^{\infty} \sum_{l=-\infty}^{\infty} j \cos ((j-l) t) p_{j} p_{l}\right| \leq E|X|,
$$

similarly to (10), we get

$$
\sum_{j=-\infty}^{\infty} \sum_{l=-\infty}^{\infty} j(j-l)^{2} p_{j} p_{l}=E X^{3}-E X^{2} E X
$$

Therefore,

$$
\left|\sum_{j=-\infty}^{\infty} \sum_{l=-\infty}^{\infty} j(j-l)^{2} p_{j} p_{l}\right| \leq 2 E|X|^{3} .
$$

Hence,

$$
\begin{aligned}
\left|A^{\prime}(t)\right| & =\left|-\sum_{j=-\infty}^{\infty} \sum_{l=-\infty}^{\infty} j(j-l) \sin ((j-l) t) p_{j} p_{l}\right| \\
& \leq\left|\sum_{j=-\infty}^{\infty} \sum_{l=-\infty}^{\infty} j(j-l)^{2} t p_{j} p_{l}\right| \\
& \leq \tau_{1}\left|\sum_{j=-\infty}^{\infty} \sum_{l=-\infty}^{\infty} j(j-l)^{2} p_{j} p_{l}\right| \\
& \leq 2 \tau_{1} E|X|^{3} \\
& \leq \frac{2}{10 \sqrt[3]{E|X|^{3}}} E|X|^{3} \\
& =\frac{1}{5}\left(E|X|^{3}\right)^{\frac{2}{3}}
\end{aligned}
$$


and

$$
\begin{aligned}
\left|A^{\prime \prime}(t)\right| & =\left|-\sum_{j=-\infty}^{\infty} \sum_{l=-\infty}^{\infty} j(j-l)^{2} \cos ((j-l) t) p_{j} p_{l}\right| \\
& \leq\left|\sum_{j=-\infty}^{\infty} \sum_{l=-\infty}^{\infty} j(j-l)^{2} p_{j} p_{l}\right| \\
& \leq 2 E|X|^{3} .
\end{aligned}
$$

By (14), we get

$$
\begin{aligned}
B(t) & =\sum_{j=-\infty}^{\infty} \sum_{l=-\infty}^{\infty} \cos ((j-l) t) p_{j} p_{l} \\
& =\sum_{j=-\infty}^{\infty} \sum_{l=-\infty}^{\infty}\left(1-2 \sin ^{2}\left(\frac{(j-l) t}{2}\right)\right) p_{j} p_{l} \\
& =\sum_{j=-\infty}^{\infty} \sum_{l=-\infty}^{\infty} p_{j} p_{l}-2 \sum_{i} \sum_{j} \sin ^{2}\left(\frac{(j-l) t}{2}\right) p_{j} p_{l} \\
& =1-2 \sum_{j=-\infty}^{\infty} \sum_{l=-\infty}^{\infty} \sin ^{2}\left(\frac{(j-l) t}{2}\right) p_{j} p_{l} \\
& \geq \frac{99}{100} .
\end{aligned}
$$

From this fact and $B(t) \leq 1$,

$$
\frac{99}{100} \leq B(t) \leq 1
$$

By (10) and (13), we obtain

$$
\begin{aligned}
\left|B^{\prime}(t)\right| & =\left|-\sum_{j=-\infty}^{\infty} \sum_{l=-\infty}^{\infty}(j-l) \sin ((j-l) t) p_{j} p_{l}\right| \\
& \leq \sum_{j=-\infty}^{\infty} \sum_{l=-\infty}^{\infty}(j-l)^{2} t p_{j} p_{l} \\
& =2 \tau_{1} \sigma^{2}(X) \\
& \leq \frac{2}{10 \sqrt[3]{E|X|^{3}}}\left(E|X|^{3}\right)^{\frac{2}{3}} \\
& =\frac{1}{5}\left(E|X|^{3}\right)^{\frac{1}{3}}
\end{aligned}
$$

and

$$
\left|B^{\prime \prime}(t)\right|=\left|-\sum_{j=-\infty}^{\infty} \sum_{l=-\infty}^{\infty}(j-l)^{2} \cos ((j-l) t) p_{j} p_{l}\right|
$$




$$
\begin{aligned}
& \leq \sum_{j=-\infty}^{\infty} \sum_{l=-\infty}^{\infty}(j-l)^{2} p_{j} p_{l} \\
& =2 \sigma^{2}(X) \\
& \leq 2\left(E|X|^{3}\right)^{\frac{2}{3}} .
\end{aligned}
$$

By (16), we obtain

$$
\theta^{(3)}(t)=\frac{(B(t))^{2} A^{\prime \prime}(t)-B(t) A(t) B^{\prime \prime}(t)-2 B^{\prime}(t) B(t) A^{\prime}(t)-2 A(t)\left(B^{\prime}(t)\right)^{2}}{(B(t))^{3}} .
$$

From this fact and (17)-(22), we get $\left|\theta^{(3)}(t)\right| \leq 4.2874 E|X|^{3}$.

\section{Proof of the main results}

Let $X_{1}, X_{2}, \ldots, X_{n}$ be independent integral-valued random variables. Let $S_{n}:=\sum_{i=1}^{n} X_{i}$, $\mu:=E S_{n}$ and $\sigma^{2}:=\operatorname{Var} S_{n}$. Let $\psi_{1}, \psi_{2}, \ldots, \psi_{n}$ and $\psi$ be the characteristic functions of $X_{1}, X_{2}, \ldots, X_{n}$ and $S_{n}$, respectively. Then, for $j=1,2, \ldots, n$,

$$
\psi_{j}(t)=\sum_{l=-\infty}^{\infty} p_{j l} e^{i l t}=\sum_{l=-\infty}^{\infty} p_{j l} \cos (l t)+i \sum_{l=-\infty}^{\infty} p_{j l} \sin (l t)
$$

and

$$
\psi(t)=\prod_{j=1}^{n} \psi_{j}(t)
$$

Note that $\psi_{j}(t)=\left|\psi_{j}(t)\right| e^{i \theta_{j}(t)}$,

where $\theta_{j}(t):=\operatorname{argument}$ of $\psi_{j}(t)=\arctan \left(\frac{\sum_{l=-\infty}^{\infty} p_{j l} \sin (l t)}{\sum_{l=-\infty}^{\infty} p_{j l} \cos (l t)}\right)$.

Hence, $\psi(t)=\rho(t) e^{i \theta(t)}$, where $\theta(t)=\sum_{j=1}^{n} \theta_{j}(t)(\bmod 2 \pi)$ and $\rho(t)=\prod_{j=1}^{n}\left|\psi_{j}(t)\right|$.

From Siripraparat and Neammanee [13], we know that

$$
P\left(S_{n}=k\right)=\frac{1}{\pi} \int_{0}^{\pi} \rho(t) \cos ((k-\mu) t-\alpha(t)) d t
$$

where $\alpha(t)=\theta(t)-\mu t$.

To prove our main theorems, we give the bound of $\rho(t)$ and $\cos ((k-\mu) t-\alpha(t))$ in Lemma 3.1 and Lemma 3.2, respectively.

Lemma 3.1 Let $\tau=\min \left(\frac{1}{\sqrt[10 \sqrt[3]{\sum_{j=1}^{n} E\left|X_{j}\right|^{3}}]{n}}, \pi\right)$. Then

$$
\left|\rho(t)-e^{-\frac{1}{2} \sigma^{2} t^{2}}\right| \leq 0.6672 \sum_{j=1}^{n} E\left|X_{j}\right|^{3} t^{3} e^{-\frac{1}{2} \sigma^{2} t^{2}} \quad \text { for } t \in[0, \tau) .
$$

Proof By Lemma 2.2 and Lemma 2.3, we get

$$
e^{-\frac{1}{2} \sigma^{2}\left(X_{j}\right) t^{2}-\frac{2}{3} E\left|X_{j}\right|^{3} t^{3}} \leq\left|\psi_{j}(t)\right| \leq e^{-\frac{1}{2} \sigma^{2}\left(X_{j}\right) t^{2}+\frac{2}{3} E\left|X_{j}\right|^{3} t^{3}} .
$$


By (24), we obtain

$$
e^{-\frac{1}{2} \sigma^{2} t^{2}-\frac{2}{3} \sum_{j=1}^{n} E\left|X_{j}\right|^{3} t^{3}} \leq \rho(t) \leq e^{-\frac{1}{2} \sigma^{2} t^{2}+\frac{2}{3} \sum_{j=1}^{n} E\left|X_{j}\right|^{3} t^{3}} .
$$

Thus,

$$
\begin{aligned}
\left(e^{-\frac{2}{3} \sum_{j=1}^{n} E\left|X_{j}\right|^{3} t^{3}}-1\right) e^{-\frac{1}{2} \sigma^{2} t^{2}} & \leq \rho(t)-e^{-\frac{1}{2} \sigma^{2} t^{2}} \\
& \leq\left(e^{\frac{2}{3} \sum_{j=1}^{n} E\left|X_{j}\right|^{3} t^{3}}-1\right) e^{-\frac{1}{2} \sigma^{2} t^{2}} .
\end{aligned}
$$

Hence,

$$
\begin{aligned}
-\frac{2}{3} \sum_{j=1}^{n} E\left|X_{j}\right|^{3} t^{3} e^{-\frac{1}{2} \sigma^{2} t^{2}} & \leq \rho(t)-e^{-\frac{1}{2} \sigma^{2} t^{2}} \\
& \leq \frac{2}{3} \sum_{j=1}^{n} E\left|X_{j}\right|^{3} t^{3} e^{\frac{2}{3} \sum_{j=1}^{n} E\left|X_{j}\right|^{3} t^{3}} e^{-\frac{1}{2} \sigma^{2} t^{2}},
\end{aligned}
$$

where we have used the fact

$$
e^{x}-1 \leq x e^{x} \text { and } e^{-x}-1>-x \text { for } x>0 .
$$

Since $t^{3} \leq \frac{1}{1000 \sum_{j=1}^{n} E\left|X_{j}\right|^{3}}$ and (25), $\left|\rho(t)-e^{-\frac{1}{2} \sigma^{2} t^{2}}\right| \leq 0.6672 \sum_{j=1}^{n} E\left|X_{j}\right|^{3} t^{3} e^{-\frac{1}{2} \sigma^{2} t^{2}}$.

Lemma 3.2 For $t \in[0, \tau]$, we have $\cos ((k-\mu) t-\alpha(t))=\cos ((k-\mu) t)+\Delta$, where $|\Delta| \leq$ $0.7152 \sum_{j=1}^{n} E\left|X_{j}\right|^{3} t^{3}$.

Proof Using Taylor's expansion, we have

$$
\begin{aligned}
& \cos (\alpha(t))=1-\frac{1}{2} \cos \left(t_{2}\right)(\alpha(t))^{2} \text { for some } t_{2}, \\
& \sin (\alpha(t))=\alpha(t)-\frac{1}{2} \sin \left(t_{3}\right)(\alpha(t))^{2} \text { for some } t_{3}, \quad \text { and } \\
& \theta_{j}(t)=\theta_{j}^{(1)}(0) t+\frac{1}{2} \theta_{j}^{(2)}(0) t^{2}+\frac{1}{6} \theta_{j}^{(3)}\left(t_{4}\right) t^{3} \quad \text { for some } t_{4} .
\end{aligned}
$$

By Lemma 2.4, (28) and the fact that $\tau \leq \tau_{1}$, we get

$$
\begin{aligned}
|\alpha(t)| & \leq \frac{1}{6} \sum_{j=1}^{n} 2.1437\left(E\left|X_{j}\right| \sigma^{2}\left(X_{j}\right)+E\left|X_{j}\right|^{3}\right) t^{3} \\
& \leq 0.7146 \sum_{j=1}^{n} E\left|X_{j}\right|^{3} t^{3} .
\end{aligned}
$$

By (26) and (27), we obtain

$$
\begin{aligned}
& \cos ((k-\mu) t-\alpha(t)) \\
& \quad=\cos ((k-\mu) t) \cos (\alpha(t))+\sin ((k-\mu) t) \sin (\alpha(t))
\end{aligned}
$$




$$
\begin{aligned}
& =\cos ((k-\mu) t)\left[1-\frac{1}{2} \cos \left(t_{2}\right) \alpha^{2}(t)\right]+\sin ((k-\mu) t)\left[\alpha(t)-\frac{1}{2} \sin \left(t_{3}\right) \alpha^{2}(t)\right] \\
& =\cos ((k-\mu) t)+\Delta
\end{aligned}
$$

where

$$
|\Delta| \leq|\alpha(t)|+\alpha^{2}(t)
$$

By (29) and $t^{3} \leq \frac{1}{1000 \sum_{j=1}^{n} E\left|X_{j}\right|^{3}}$, we obtain $|\alpha(t)| \leq \frac{0.7146}{1000}$.

From this fact, (29) and (30) imply that $|\triangle| \leq 0.7152 \sum_{j=1}^{n} E\left|X_{j}\right|^{3} t^{3}$.

We are now ready to prove Theorem 1.2.

Proof of Theorem 1.2 Note that

$$
\frac{1}{\pi} \int_{0}^{\pi} \rho(t) \cos ((k-\mu) t-\alpha(t)) d t=\frac{1}{\pi} \int_{0}^{\tau} \rho(t) \cos ((k-\mu) t-\alpha(t)) d t+\triangle_{1},
$$

where $\triangle_{1}=\frac{1}{\pi} \int_{\tau}^{\pi} \rho(t) \cos ((k-\mu) t-\alpha(t)) d t$.

By Lemma 2.1, $\left|\triangle_{1}\right| \leq \frac{1}{\pi} \int_{\tau}^{\pi} \rho(t) d t \leq \frac{1}{\pi} \int_{\tau}^{\infty} e^{-\frac{1}{\pi^{2}} \alpha t^{2}} d t \leq \frac{\pi}{2 \tau \alpha} e^{-\frac{\tau^{2} \alpha}{\pi^{2}}}$.

From the fact that

$$
\int_{0}^{\infty} t^{3} e^{-\frac{1}{2} \sigma^{2} t^{2}} d t=\frac{2}{\sigma^{4}}
$$

and Lemma 3.1, we have

$$
\frac{1}{\pi} \int_{0}^{\tau} \rho(t) \cos ((k-\mu) t-\alpha(t)) d t=\frac{1}{\pi} \int_{0}^{\tau} e^{-\frac{1}{2} \sigma^{2} t^{2}} \cos ((k-\mu) t-\alpha(t)) d t+\triangle_{2},
$$

where

$$
\begin{aligned}
\left|\triangle_{2}\right| & \leq 0.6672 \sum_{j=1}^{n} E\left|X_{j}\right|^{3} \int_{0}^{\tau} t^{3} e^{-\frac{1}{2} \sigma^{2} t^{2}} d t \\
& \leq 0.6672 \sum_{j=1}^{n} E\left|X_{j}\right|^{3} \int_{0}^{\infty} t^{3} e^{-\frac{1}{2} \sigma^{2} t^{2}} d t \\
& =\frac{1.3344}{\sigma^{4}} \sum_{j=1}^{n} E\left|X_{j}\right|^{3} .
\end{aligned}
$$

From (32) and Lemma 3.2, we get

$$
\frac{1}{\pi} \int_{0}^{\tau} e^{-\frac{1}{2} \sigma^{2} t^{2}} \cos ((k-\mu) t-\alpha(t)) d t=\frac{1}{\pi} \int_{0}^{\tau} e^{-\frac{1}{2} \sigma^{2} t^{2}} \cos ((k-\mu) t) d t+\triangle_{3},
$$

where

$$
\left|\triangle_{3}\right| \leq \frac{0.7152}{\pi} \sum_{j=1}^{n} E\left|X_{j}\right|^{3} \int_{0}^{\infty} t^{3} e^{-\frac{1}{2} \sigma^{2} t^{2}} d t=\frac{0.4554}{\sigma^{4}} \sum_{j=1}^{n} E\left|X_{j}\right|^{3} .
$$


By(31) and (33)-(36), we obtain

$$
\frac{1}{\pi} \int_{0}^{\pi} \rho(t) \cos ((k-\mu) t-\alpha(t)) d t=\frac{1}{\pi} \int_{0}^{\tau} e^{-\frac{1}{2} \sigma^{2} t^{2}} \cos ((k-\mu) t) d t+\Delta_{4},
$$

where

$$
\begin{aligned}
\left|\triangle_{4}\right| & \leq\left|\triangle_{1}\right|+\left|\triangle_{2}\right|+\left|\triangle_{3}\right| \\
& \leq \frac{\pi}{2 \tau \alpha} e^{-\frac{\tau^{2} \alpha}{\pi^{2}}}+\frac{1.3344}{\sigma^{4}} \sum_{j=1}^{n} E\left|X_{j}\right|^{3}+\frac{0.4554}{\sigma^{4}} \sum_{j=1}^{n} E\left|X_{j}\right|^{3} \\
& =\frac{\pi}{2 \tau \alpha} e^{-\frac{\tau^{2} \alpha}{\pi^{2}}}+\frac{1.7898}{\sigma^{4}} \sum_{j=1}^{n} E\left|X_{j}\right|^{3} .
\end{aligned}
$$

From (10), we can see that

$$
\alpha=2 \sum_{j=1}^{n} \sum_{l=-\infty}^{\infty} p_{j l} p_{j(l+1)}=\sum_{j=1}^{n}\left(\sum_{\substack{l=-\infty \\|l-m| \leq 1}}^{\infty} \sum_{m=-\infty}^{\infty}(l-m)^{2} p_{j l} p_{j m}\right) \leq 2 \sigma^{2}
$$

which implies that $e^{-\frac{1}{2} \sigma^{2} t^{2}} \leq e^{-\frac{1}{4} \alpha t^{2}}$. From this fact, we get

$$
\begin{aligned}
\frac{1}{\pi}\left|\int_{\tau}^{\infty} e^{-\frac{1}{2} \sigma^{2} t^{2}} \cos ((k-\mu) t) d t\right| & \leq \frac{1}{\pi} \int_{\tau}^{\infty} e^{-\frac{1}{2} \sigma^{2} t^{2}} d t \\
& \leq \frac{1}{\pi} \int_{\tau}^{\infty} e^{-\frac{1}{4} \alpha t^{2}} d t \\
& \leq \frac{1}{\pi \tau} \int_{\tau}^{\infty} t e^{-\frac{1}{4} \alpha t^{2}} d t \\
& =\frac{2}{\pi \tau \alpha} e^{-\frac{\tau^{2} \alpha}{4}} .
\end{aligned}
$$

From this fact and (37) and (38), we have

$$
\frac{1}{\pi} \int_{0}^{\pi} \rho(t) \cos ((k-\mu) t-\alpha(t)) d t=\frac{1}{\pi} \int_{0}^{\infty} e^{-\frac{1}{2} \sigma^{2} t^{2}} \cos ((k-\mu) t) d t+\Delta_{5},
$$

where

$$
\begin{aligned}
\left|\triangle_{5}\right| & \leq\left|\triangle_{4}\right|+\frac{1}{\pi}\left|\int_{\tau}^{\infty} e^{-\frac{1}{2} \sigma^{2} t^{2}} \cos ((k-\mu) t) d t\right| \\
& \leq \frac{\pi}{2 \tau \alpha} e^{-\frac{\tau^{2} \alpha}{\pi^{2}}}+\frac{1.7898}{\sigma^{4}} \sum_{j=1}^{n} E\left|X_{j}\right|^{3}+\frac{2}{\pi \tau \alpha} e^{-\frac{\tau^{2} \alpha}{4}} .
\end{aligned}
$$

Using the fact that

$$
\int_{0}^{\infty} e^{-a t^{2}} \cos (b t) d t=\frac{1}{2} \sqrt{\frac{\pi}{a}} e^{-\frac{b^{2}}{4 a}} \quad \text { for } a>0
$$


(see [13], p. 7), we obtain

$$
\frac{1}{\pi} \int_{0}^{\infty} e^{-\frac{1}{2} \sigma^{2} t^{2}} \cos ((k-\mu) t) d t=\frac{1}{\sigma \sqrt{2 \pi}} e^{-\frac{(k-\mu)^{2}}{2 \sigma^{2}}}
$$

By (23), (39), (40), and (41), we can conclude that

$$
P\left(S_{n}=k\right)=\frac{1}{\sigma \sqrt{2 \pi}} e^{-\frac{(k-\mu)^{2}}{2 \sigma^{2}}}+\triangle_{6},
$$

where $\left|\triangle_{6}\right| \leq \frac{2.2075 e^{-\frac{\tau^{2} \alpha}{\pi^{2}}}}{\tau \alpha}+\frac{1.7898}{\sigma^{4}} \sum_{j=1}^{n} E\left|X_{j}\right|^{3}$.

Proof of Theorem 1.3 Let $Y_{j}=\frac{X_{j}}{b}-\frac{a}{b}$. Then

$$
\begin{aligned}
& E\left(\sum_{j=1}^{n} Y_{j}\right)=\frac{\mu-n a}{b}, \quad \operatorname{Var}\left(\sum_{j=1}^{n} Y_{j}\right)=\frac{\sigma^{2}}{b^{2}} \\
& P\left(S_{n}=n a+k b\right)=P\left(\sum_{j=1}^{n} Y_{j}=k\right)
\end{aligned}
$$

and

$$
P\left(Y_{j}=k\right)=P\left(\frac{X_{j}}{b}-\frac{a}{b}=k\right)=P\left(X_{j}=a+b k\right)
$$

Since $b$ is maximal, we have $\alpha=\sum_{j=1}^{n} \alpha_{j}>0$,

where $\alpha_{j}=2 \sum_{l=-\infty}^{\infty} p_{j l} p_{j(l+1)}, p_{j l}=P\left(X_{j}=a+b l\right)$.

From Theorem 1.2, we obtain Theorem 1.3.

\section{Examples of the main results}

In this section, we give applications including Poisson binomial, binomial, and negative binomial that our main theorems can be applied as shown in Example 1-Example 3. In addition, the example that our main results can be applied to, unlike the result of Petrov [1], as shown in Example 4.

Example 1 If $X_{1}, X_{2}, \ldots, X_{n}$ are independent Bernoulli random variables with $P\left(X_{j}=1\right)=$ $p_{j}$ and $P\left(X_{j}=0\right)=q_{j}$, where $p_{j}+q_{j}=1$ for $j=1,2, \ldots, n, S_{n}$ is a Poisson binomial random variable. Then

$$
\left|P\left(S_{n}=k\right)-\frac{1}{\sigma \sqrt{2 \pi}} e^{-\frac{(k-\mu)^{2}}{2 \sigma^{2}}}\right| \leq \frac{11.0375 \sqrt[3]{\mu} e^{-\frac{\sigma^{2}}{50 \pi^{2}(\sqrt[3]{\mu})^{2}}}}{\sigma^{2}}+\frac{1.7898 \mu}{\sigma^{4}}
$$

where $\mu=\sum_{j=1}^{n} p_{j}$ and $\sigma^{2}=\sum_{j=1}^{n} p_{j} q_{j}$

Proof Note that $E\left|X_{j}\right|^{3}=p_{j}$ and

$$
\alpha=\sum_{j=1}^{n} \alpha_{j}=2 \sum_{j=1}^{n} \sum_{l=0}^{1} p_{j, l} p_{j, l+1}
$$




$$
\begin{aligned}
& =2 \sum_{j=1}^{n} \sum_{l=0}^{1} P\left(X_{j}=l\right) P\left(X_{j}=l+1\right) \\
& =2 \sum_{j=1}^{n} P\left(X_{j}=0\right) P\left(X_{j}=1\right) \\
& =2 \sum_{j=1}^{n} p_{j} q_{j} \\
& =2 \sigma^{2} .
\end{aligned}
$$

Hence, by Theorem 1.2, we see that (42) holds.

Example 2 Let $S_{n} \sim \operatorname{Bi}(p)$. Then

$$
\left|P\left(S_{n}=k\right)-\frac{1}{\sqrt{2 \pi n p q}} e^{-\frac{(k-n p)^{2}}{2 n p q}}\right| \leq \frac{11.0375 \sqrt[3]{p} e^{-\frac{n p q}{50 \pi^{2}\left(\sqrt[3]{n p)^{2}}\right.}}}{n^{\frac{2}{3}} p q}+\frac{1.7898}{n p q^{2}} .
$$

Proof We can apply Example 1 by letting $p_{j}=p$ and $q_{j}=q$.

Observe that the results in Example 1 and Example 2 have the same order as (3) and (4) but the constants are bigger. However, (3) and (4) cannot be applied with the following example.

Example 3 If $X_{1}, X_{2}, \ldots, X_{n}$ are i.i.d. geometric random variables with parameter $p$. Then

$$
\begin{aligned}
& \left|P\left(S_{n}=k\right)-\frac{p}{\sqrt{2 \pi q}} e^{-\frac{(k p-1)^{2}}{2 q}}\right| \\
& \quad \leq \frac{11.0375(1+q) \sqrt[3]{p^{2}+6 q} e^{-\frac{n p^{3} q}{50 \pi^{2}(1+q)\left(\sqrt[3]{\left.n\left(p^{2}+6 q\right)\right)^{2}}\right.}}}{n^{\frac{2}{3}} p^{2} q}+\frac{1.7898 p\left(p^{2}+6 q\right)}{n q^{2}},
\end{aligned}
$$

where $q=1-p$.

Proof Let $\psi$ be the characteristic function of $X_{j}$. Then $\psi(t)=\frac{p e^{i t}}{1-q e^{i t}}$ and

$$
\psi^{(3)}(t)=-\frac{i p e^{i t}\left(q^{2} e^{2 i t}+4 q e^{i t}+1\right)}{\left(1-q e^{i t}\right)^{4}} .
$$

Hence, $E X^{3}=\frac{\psi^{(3)}(0)}{i^{3}}=\frac{p^{2}+6 q}{p^{3}}$.

Note that

$$
\begin{aligned}
\alpha & =2 n \sum_{l=1}^{\infty} p_{1, l} p_{1, l+1}=2 n \sum_{l=1}^{\infty} P\left(X_{1}=l\right) P\left(X_{1}=l+1\right) \\
& =2 n \frac{p^{2}}{q} \sum_{l=1}^{\infty} q^{2 l} \\
& =\frac{2 n p q}{1+q}
\end{aligned}
$$

Hence, by Theorem 1.4, we get (43). 
Example 4 Let $X_{n}$ be a sequence of independent random variables such that

$$
P\left(X_{j}=0\right)=\frac{1}{4}, P\left(X_{j}=1\right)=\frac{3}{8} \quad \text { and } \quad P\left(X_{j}=2\right)=\frac{3}{8}
$$

for all $j=1,2, \ldots, n$. Then

$$
\left|P\left(S_{n}=k\right)-\frac{0.5111}{\sqrt{n}} e^{-\frac{(8 k-9 n)^{2}}{72 n}}\right| \leq \frac{0.069}{n^{\frac{2}{3}}} e^{-0.00022 n^{\frac{1}{3}}}+\frac{16.2671}{n} .
$$

Proof Note that $E\left|X_{j}\right|^{3}=\frac{27}{8}, E S_{n}=\frac{9 n}{8}, \operatorname{Var} S_{n}=\frac{39 n}{64}$, and

$$
\begin{aligned}
\alpha & =\sum_{j=1}^{n} \alpha_{j}=2 \sum_{j=1}^{n} \sum_{l=0}^{2} p_{j, l} p_{j, l+1} \\
& =2 \sum_{j=1}^{n} \sum_{l=0}^{2} P\left(X_{j}=l\right) P\left(X_{j}=l+1\right) \\
& =2 \sum_{j=1}^{n}\left(P\left(X_{j}=0\right) P\left(X_{j}=1\right)+P\left(X_{j}=1\right) P\left(X_{j}=2\right)\right) \\
& =2 \sum_{j=1}^{n}\left(\frac{1}{4} \times \frac{3}{8}+\frac{3}{8} \times \frac{3}{8}\right) \\
& =\frac{15 n}{32} .
\end{aligned}
$$

Hence, by Theorem 1.2, we see that (44) holds.

One can see that Theorem 1.2 can be applied to Example 4 and get the rate of convergence $O\left(\frac{1}{n}\right)$, but Petrov's theorem [1] cannot be applied because this example does not satisfy its assumption 3.

\section{Acknowledgements}

The authors would like to thank the reviewers for their valuable comments and suggestions.

\section{Funding}

This work was supported by the Development and Promotion of Science and Technology Talents Project (DPST).

Availability of data and materials

Not applicable.

\section{Competing interests}

The authors declare that they have no competing interests.

Authors' contributions

The authors contributed equally in writing the final version of this article. All authors read and approved the final manuscript.

\section{Publisher's Note}

Springer Nature remains neutral with regard to jurisdictional claims in published maps and institutional affiliations. Received: 26 August 2020 Accepted: 18 March 2021 Published online: 25 March 2021 


\section{References}

1. Petrov, V.V.: Sums of Independent Random Variables. Springer, New York (1975). Translated from the Russian by A.A. Brown, Ergebnisse der Mathematik und ihrer Grenzgebiete, Band 82

2. Giuliano Antonini, R., Weber, M.: Approximate local limit theorems with effective rate and application to random walks in random scenery. Bernoulli 23(4B), 3268-3310 (2017)

3. Berry, A.C.: The accuracy of the Gaussian approximation to the sum of independent variables. Transl. Am. Math. Soc. 49, 122-136 (1941)

4. Esseen, C.G.: On the Liapounoff limit of error in the theory of probability. Ark. Mat. Astron. Fys. 28A, 1-19 (1942)

5. Shevtsova, I.G.: An improvement of convergence rate estimates in the Lyapunov theorem. Dokl. Math. 82(3), 862-864 (2010)

6. Shevtsova, I.G.: Moment-type estimates with an improved structure for the accuracy of the normal approximation to distributions of sums of independent symmetric random variables. Teor. Veroâtn. Primen. 57, 499-532 (2012) (Russian). English transl. Theory Probab. Appl. 57, 468-496 (2013)

7. Shiganov, I.S.: A refinement of the upper bound of the constant in the remainder term of the central limit theorem. J. Sov. Math. 3, 2545-2550 (1986)

8. Shevtsova, I.G.: On the absolute constants in the Berry-Esseen inequality and its structural and nonuniform improvements. Inform. Primen. 7(1), 124-125 (2013) (Russian)

9. Tyurin, I.: A refinement of the remainder in the Lyapunov theorem. Theory Probab. Appl. 56(4), 693-696 (2010)

10. Van Beeck, P.: An application of Fourier methods to the problem of sharpening the Berry-Esseen inequality. Z. Wahrscheinlichkeitstheor. Verw. Geb. 23, 187-196 (1972)

11. McDonald, D.R.: The local limit theorem: a historical perspective. JIRSS 4(2), 73-86 (2005)

12. Zolotukhin, A., Nagaev, S., Chebotarev, V.: On a bound of the absolute constant in the Berry-Esseen inequality for i.i.d. Bernoulli random variables. Mod. Stoch. Theory Appl. 5(3), 385-410 (2018)

13. Siripraparat, T., Neammanee, K.: A local limit theorem for Poisson binomial random variable. Sci. Asia. https://doi.org/10.2306/scienceasia1513-1874.2021.006

14. Doob, J.L.: Stochastic Processes. Wiley, New York (1953)

15. Prokhorov, Yu.V., Rozanov, Yu.A.: Probability Theory. Nauka, Moscow (1973) (in Russian)

16. Statulevichus, V.A.: Limit theorems for densities and asymptotic decompositions for distributions of sums of independent random variables. Teor. Veroâtn. Primen. 10(4), 645-659 (1965)

17. Ushakov, N.G.: Lower and upper bounds for characteristic functions. J. Math. Sci. 84, 1179-1189 (1997)

18. Ushakov, N.G.: Selected Topics in Characteristic Functions. VSP, Utrecht (1999)

19. Benedicks, M.: An estimate of the modulus of the characteristic function of a lattice distribution with application to remainder term estimates in local limit theorems. Ann. Probab. 3, 162-165 (1975)

20. Zhang, Z:: An upper bound for characteristic functions of lattice distributions with applications to survival probabilities of quantum states. J. Phys. A, Math. Theor. 40, 131-137 (2007)

21. Zhang, Z: Bounds for characteristic functions and Laplace transforms of probability distributions. Theory Probab. Appl. 56(2), 350-358 (2012)

22. Neammanee, K.: A refinement of normal approximation to Poisson binomial. Int. J. Math. Math. Sci. 5, 717-728 (2005)

\section{Submit your manuscript to a SpringerOpen ${ }^{\circ}$ journal and benefit from:}

- Convenient online submission

- Rigorous peer review

- Open access: articles freely available online

- High visibility within the field

- Retaining the copyright to your article

Submit your next manuscript at $\boldsymbol{s p r i n g e r o p e n . c o m ~}$ 